\title{
The multi-depot electric vehicle location routing problem with time windows
}

\author{
Juan Camilo Paz ${ }^{\mathrm{a}}$, Mauricio Granada-Echeverria and John Willmer Escobar ${ }^{\mathrm{b}}{ }^{\text {* }}$
}

${ }^{a}$ Faculty of Electrical Engineering, Universidad Tecnológica de Pereira, Pereira, Colombia ${ }^{b}$ Department of Accounting and Finance, Universidad del Valle, Cali, Colombia

\begin{tabular}{l}
\hline C H R O N I C L E \\
\hline Article history: \\
Received October 272016 \\
Received in Revised Format \\
December 222016 \\
Accepted February 272017 \\
Available online \\
March 1 2017 \\
\hline Keywords: \\
Multi-depot \\
Electric vehicle \\
Vehicle location routing problem \\
Time windows
\end{tabular}

Time windows

\begin{abstract}
A B S T R A C T
In this paper, the Multi-Depot Electric Vehicle Location Routing Problem with Time Windows (MDVLRP) is addressed. This problem is an extension of the MDVLRP, where electric vehicles are used instead of internal combustion engine vehicles. The recent development of this model is explained by the advantages of this technology, such as the diminution of carbon dioxide emissions, and the support that they can provide to the design of the logistic and energy-support structure of electric vehicle fleets. There are many models that extend the classical VRP model to take electric vehicles into consideration, but the multi-depot case for location-routing models has not been worked out yet. Moreover, we consider the availability of two energy supply technologies: the "Plug-in" Conventional Charge technology, and Battery Swapping Stations; options in which the recharging time is a function of the amount of energy to charge and a fixed time, respectively. Three models are proposed: one for each of the technologies mentioned above, and another in which both options are taken in consideration. The models were solved for small scale instances using $\mathrm{C}++$ and Cplex 12.5. The results show that the models can be used to design logistic and energy-support structures, and compare the performance of the different options of energy supply, as well as measure the impact of these decisions on the overall distance traveled or other optimization objectives that could be worked on in the future.
\end{abstract}

\section{Introduction}

Electric Vehicles (EVs) are a transportation alternative of interest to governments, academics, and companies. This can be explained by the fact that EVs can mitigate greenhouse gas emissions, and even eliminate them if the energy consumed comes from renewable sources; therefore, multiple efforts have been made to achieve the implementation of this technology in the future, such as the testing of real sector companies (UPS, 2013, DPDHL, 2014). However, to achieve the successful implementation of this technology, one of the challenges to solve is the design of the energy supply network that supports this system. In addition, if you plan your design with tools such as mathematical modeling or metaheuristics, you could optimize the system's operation. One way to do this is to use some of the mentioned techniques to determine design aspects such as the location and type of recharging needed.

\footnotetext{
* Corresponding author Tel: +57 3005349187

E-mail: john.wilmer.escobar@correounivalle.edu.co (J.W. Escobar)

(C) 2018 Growing Science Ltd. All rights reserved. doi: $10.5267 /$ j.ijiec.2017.4.001
} 
In summary, the technological benefits of EVs, the need to mitigate the unfavorable effects of human activity on the environment, and the potential of the decision-support models on the design of the operational configuration required for the EVs justifies the development of models that allow the optimal designing of these systems.

For that reason in this article, a mathematical approach is developed that allows the designing of the operative logistic network of a homogenous EV fleet, determining: i) the number and location of the recharging station, ii) the number and location of the depots, and iii) the number of vehicles and their routes of operation. Two types of technologies are considered: i) the charge of the battery by direct supply of the energy net, and ii) the use of Battery Swapping Stations (BSS). Moreover, several constraints are considered such as time windows and the autonomy and freight capacity of the EVs. However, the existence of different types of recharging strategy implies different ways of modeling. For this reason, three mixed integer linear programing models are proposed in order to support the design decisions that allow a performance comparison between the recharging strategies. These models are defined as follows: i) the conventional charge case, in which not only does the special charge vertex have this technology but instead, the customer and depot nodes are allowed to have it too. Furthermore, the charge of the battery could be partial or complete; and ii) in the case of the BSS, the location of the recharging stations is only possible at special charge vertices due to the complex structure required for the battery swapping that makes the application of this technology at customer vertices impossible. Additionally, each depot has the possibility to install a BSS. The third model is defined in a way that if a charging vertex is activated, then it is a BSS but if a customer vertex is activated for recharging, it is of conventional recharging.

In order to achieve the objectives set out in this investigation, a review was made of the already developed models that support the design of combustion vehicle operating systems. It was found that these models are not immediately extendible to the case of EVs, since they have special characteristics such as their limited autonomy, the possibility of using multiple charge technologies, and the need for an energy supply structure. For example, the autonomy of the vehicle and the location of the charging stations affect the routing decisions. This happens when an EV needs to be charged and therefore, is forced to use part of its operating time in moving to a charging station (time that is influenced by the location of the stations); aspects to consider at the time of determining a route, since the time window constraints of the customers must be met. Similarly, the location decisions of the recharging stations are affected by the defined routes because they are the demand indicator for that decision. From this explanation, it is clear that routing and location decisions are dependent on each other and it can be inferred that, when taken together, it is possible to improve the optimization objective more than if these decisions are taken independently, which justifies the approach of determining the location and routing decisions at the same time. This applies to different optimization objectives, such as the total distance traveled, the number of recharging facilities, and the number of vehicles used, as shown in the work of Schiffer and Walther (2017).

As explained, the characteristics of the EVs do not allow an immediate application of the literature models oriented to combustion vehicles to that case. And although several models have already been developed to extend the literature on the Vehicle Location Routing Problem with the particularities of EVs, in the literature, this problem has not been extended with EVs to the case of multiple depots, in this case addressed by the authors and defined as the Multi-Depot Electric Vehicle Location Routing Problem with Time Windows (MDEVLRPTW). The model is an extension of the Multi Depot Vehicle Location Routing Problem with Time Windows in which EVs are used instead of combustion vehicles.

For this reason, three models are defined in this research that differ according to the battery charging strategy. Consider $R$ as the set of vertices where conventional charging stations or BSS can be located, and $C$ as the set of customer vertices. Then the proposed models are: 
- Multi Depot Electric Vehicle Location Routing Problem with Time Windows and Battery Swapping (MDEVLRPTW-BS). It is assumed in this case that only BSS can be located at vertex $i \in R$.

- Multi Depot Electric Vehicle Location Routing Problem with Time Windows and Partial Recharging (MDEVLRPTW-PR). It is assumed in this case that conventional charging stations can be located at the vertex $i \in(C \cup R)$. The charge can be partial or complete. Charge of battery is considered at depots by defining vertex $i \in R$ with the same coordinates as the depots.

- Multi Depot Electric Vehicle Location Routing Problem with Time Windows Battery Swapping and Partial Recharging (MDEVLRPTW-BSPR). It is assumed in this case that if a charging vertex $i \in R$ is activated it is a BSS, while if it is activated a vertex $i \in C$ it is a conventional recharging point.

All the models are aimed at minimizing the total distance traveled; however, the proposed mathematical structures allow to minimize the number of vehicles given a fixed number of charging stations (conventional or BSS) as well as minimizing the number of charging stations given a fixed number of vehicles.

The results of this optimization goal as well as the most relevant decisions of the three models are presented and compared using a modification of the instances of Schiffer and Walther (2017). The costs of installation are not considered in the models as an optimization objective because the instances of the literature used to validate the models do not present this information. However, the extension of the models with this feature can be easily extended for practical applications based on the present work.

The contributions of this study can be summarized as follows:

- We extend the MDVLRP to an Electric Vehicle scheme for strategic planning of electric logistic fleets.

- We define a mathematical approach to solve the problem with already well-known aspects of the literature such as partial charging at every node of the network, as well as battery exchange in specialized charging stations.

- We validate the proposed approach using a modification of the EVLRPTW instances of Schiffer and Walther (2017).

- We show that the models can be used to design small-sized logistic and energy-support structures.

This paper is structured as follows: in section 2, a literature review is given; in Section 3, the experimental methodology proposed for the problem development is presented, in which the three models are described; finally, computational results and conclusions are presented in section 4 and 5, respectively.

\section{Literature review}

One field of the literature concerned with the problem addressed in this paper is the Electric Vehicle Routing Problem (EVRP, an extension of the Vehicle Routing Problem, VRP, but adapted to EVs. A literature review of this field is presented in the work of Afroditi et al., 2014). The other is the Recharging Structure Location Problem (a general literature review can be consulted in the paper of Touati-Moungla and Jost, 2012). These aspects together compose the Location-Routing Problem. An extensive review of this problem for internal combustion engine vehicles can be consulted in the work of Prodhon and Prins (2014), and Drexl and Schneider (2014, 2015). 
In relation to EVRP approaches, one of the first works of relevance was the one presented by Conrad and Figliozzi (2011), entitled by the authors as the Recharging Vehicle Routing Problem, in which the consumption of EVs is considered. Two independent optimization objectives are defined: the minimization of the number of vehicles, and the overall costs. The charge of the vehicle only is possible at customer vertices. Then, in the work of Erdogan and Miller-Hooks (2012), specific vertices were considered for recharging stations-a model well-known as the Green Vehicle Routing Problem-work in which the minimization of the overall distance is considered. Meanwhile, Omidvar and TavakkoliMoghaddam (2012) extended the problem considering time-windows constraints and proposed a mathematical model that minimizes the costs of distance traveled, travel time and emissions. They used the Simulated Annealing (SA) and Genetic Algorithm (GA) approaches.

Barco et al. (2013) proposed an EVRP in which the total energy used is minimized. The authors used an approximate energy consumption model based on physical dependencies to compute energy consumption. The charge is only possible at the depot and the wear of the battery over time is considered. Schneider et al. (2014) developed an extension of the EVRP with time window constraints (EVRP-TW); the model focuses on EVs with route recharge options. The optimization objective used is hierarchical, in which the number of vehicles used to achieve all the customers' demands is minimized, and then the total distance traveled is minimized. This work was then extended by Hiermann et al. (2016) considering a heterogeneous fleet of EVs. The optimization objective was the overall distance and the acquisition costs of the vehicles. Desaulniers et al. (2016) worked on the same problem, but considering four recharging strategies (single-FR, single-PR, multiple-FR, and multiple-PR). The problems were tried to solve to optimality using branch-price-and-cut algorithms.

On the other hand, Goeke and Schneider (2015) extended the EVRP-TW considering a mixed fleet with EVs and combustion vehicles. And in addition, they used realistic energy consumption functions, which consider vehicle speed, vehicle mass, and terrain conditions. The authors considered as an optimization objective, the minimization of total distance traveled, energy, salary costs and a third objective with these two same costs and also the costs associated with the life of the battery. Another work that uses nonlinear functions for the EVRP is the one proposed by Montoya et al. (2016). Up to this point, all the works discussed only consider the complete charge of the battery at specialized vertices. In respect to this aspect, the work of Felipe et al. (2014) on the Green Vehicle Routing Problem with Multiple Technologies and Partial Recharges extended the literature of EVRP considering partial charging. Keskin and Çatay (2016), extended EVRPTW to a partial recharge scheme and presented the mathematical programming formulation of the problem. The authors proposed an effective ALNS method to solve the EVRPTW and EVRPTW-PR. Then, Ding et al. (2015) considered partial charge and also the capacity of the recharging stations; however, these last two jobs do not consider charging at customer vertices.

On the other hand, the recharging stations location problem has also been approached independently. One of the first works that considered the technology of BSS in this type of model was presented by Wang (2008), but postulated for electric scooters. This line was extended to the case of EVs but locating conventional charging stations instead of BSS (Wang and Lin, 2009). Then, the location of this same type of station was applied to the case of passenger vehicles (Wang and Wang, 2010). Another extension is the one proposed by Cavadas et al. (2015), in which for the location of recharging stations is considered so that the demands of the customers can be transferred between one station and another.

One of the first approach of the location-routing problem of EVs was presented by Worley and Klabjan (2012). The objective of the model is to minimize the sum of the travel costs, recharging costs, and costs of locating recharging stations, although, a solution method was not proposed. Yang and Sun (2015) propose a model that addresses both decisions, but limited to the BSS technology. The authors proposed two heuristic algorithms to solve even large-scale instances. Another work that incorporates both types of decision is the one proposed by Li-ying and Yuan-bin (2015). These authors consider that each station may have different types of technology. The type of station depends on the time of charge of the battery 
(linear function that depends on the type of station and of the amount of energy remaining), the cost of construction, and the cost of the electricity. Apart from this, they considered recharging at customer vertices by postulating vertices available for the location of recharging stations with the same customer coordinates, and also considered time window constraints. In their work, it is assumed that the battery is always fully charged. Also, a hybrid heuristic that incorporates an adaptive variable neighborhood search (AVNS) with the tabu search algorithm for intensification was used to solve small and large-scale instances. Schiffer and Walther (2017) proposed a location-routing model in which only conventional charging stations are considered but extend the mathematical formulation to consider partial charge of the battery. The model can only be solved in efficient computational times in small scale instances. Then, the same authors proposed a large-scale solution algorithm called Adaptive Large Neighborhood Search for the Location Routing Problem with Intraroute Facilities (Schiffer and Walther, 2016). The algorithm was validated by solving the proposed instances in the works of Yang and Sun (2015), and Schiffer and Walther (2017).

In relation to the literature review, the research presented in this paper extends the state-of-the-art location-routing models of EV by proposing an extension for the case of multiple depots. All this, maintaining important already well-known aspects of the literature such as time window constraints, the possibility of partial charging, the modeling of multiple visits to the vertices, the possibility of locating stations at all vertices (including depots) and also, considering two approaches of recharging the battery: the conventional charge (linear dependent on amount of energy remaining), and BSS (fixed charge time). A mathematical model is proposed for each approach as well as one in which both are considered at the same time. This allows the design of a logistics network that supports an EV fleet and allows a comparison of the linear and fixed-time charge results. For the validation of the model, we propose a set of instances based on Schiffer and Walther (2017), in which a depot is randomly added.

\section{Experimental methodology}

In this section, we present the characteristics and assumptions shared by the models proposed. The mathematical model for the MDEVLRPTW-BS is described as well as for the models MDEVLRPTWPR and MDEVLRPTW-BSPR. All the models are of mixed linear programming.

\section{Characteristics and Assumptions}

The MDEVLRPTW can be formally defined as a directed and complete graph $G$ composed of a set of vertices $V_{0+1}$ and a set of $\operatorname{arcs} A=\left\{(i, j) \mid i, j \in V_{0+1}, i \neq j\right\}$. The problem has the following characteristics:

- The set of vertices $V_{0+1}$ corresponds to the union of the set of vertices of customers to be visited $C$, the set of vertices where specialized battery recharging stations can be located $R$, the set of vertices of the depots for dispatch of vehicles $U D_{0}$, the set of vertices of the depots for vehicle arrivals $U D_{+1}\left(U D=U D_{0} \cup U D_{+1}\right)$, and a set of dummy vertices $S$ associated to the vertices $h \in$ $(C \cup R \cup U D)$. This set is introduced in order to allow multiple visits to the vertices in the construction of the routes; a feature necessary to also model the possibility of recharging multiple vehicles at the same time. (The set $S$ is the union of all the dummy vertices subsets $S K_{h}$, where $S K_{h}$ is the set of dummy vertices associated to vertex $h$ ).

- For each arc $(i, j)$ a distance $d_{i j}$ between vertex $i$ and $j$ is given.

- The travel time $t_{i j}$ for the arc $(i, j)$ is calculated as the average velocity $v$ multiplied by $d_{i j}$.

- Each vertex $i \in C$ must be visited by a vehicle to deliver the goods $p_{i}$ in the service time $s_{i}$, and this visit is made at time $t_{i}$ within a time window defined by the earliest time $e_{i}$ and the latest time $l_{i}$. 
- Each depot can be activated and from it, must exit and enter the same number of arcs. Each of the exit arcs represents a vehicle that is dispatched. These vehicles are homogeneous and have a freight capacity $D$ and a battery capacity $Q$.

- Each vehicle dispatched must leave and return to the same depot.

- The displacement of vehicles is limited by the capacity of the battery and the fixed rate of energy consumption per distance unit $c$.

- A maximum number of recharge points can be located and these can be located at the vertices $i \in(C \cup R)$. The recharge points located in any $i \in C$ are always of conventional charge. While the $i \in R$ can be conventional charge or BSS depending on the approach being treated.

- If the battery is charged by the conventional manner at some vertex, this is done at the constant charge rate of energy per unit time $r$. If a battery change is made in a BSS, this is done in a constant amount of time $c t$ which is always a smaller amount of $r$ multiplied by $Q$ (complete conventional charge time).

- If the battery is charged at a vertex $i \in C$, it is performed in parallel to the service time $s_{i}$, which allows for better optimization of the operational times.

- The problem is determinate: i) the number and location of the recharging station, ii) the number and location of the depots and iii) the number of vehicles and their routes of operation of the system to minimize the total distance traveled.

- Vehicle speed $v$ is constant and is not affected by altitude.

- Energy consumption is modeled as a linear function of the distance traveled.

- The charge time at conventional stations depends linearly on the amount of energy to be charged.

- In general, it is considered that the batteries can be charged at all the vertices of the network. Punctually, in the case of the MDEVLRPTW-BS only BSS can be used and these are located at vertices $i \in R$. In the case of the MDEVLRPTW-PR, the battery can be charged in a conventional manner at the vertices $i \in(C \cup R)$, partially or completely. Charge at depots is considered by defining vertices $i \in R$ with the same coordinates as the depots. Finally, in the case of the MDEVLRPTW-BSPR if a recharge point is activated at the vertices $i \in R$, it is of type BSS, while at a vertex $i \in C$, it is a conventional recharge point.

- The number of vehicles that can be charged at the same time at a vertex is not limited.

- If a vehicle is charged at a customer vertex, this operation is performed in parallel with the service time $s$.

- All vehicles start their route with their battery fully charged, so there is a station for overnight charge in all depots.

- Each depot is represented with two vertices that have the same coordinates: one to represent the dispatch of vehicles, and the other for arrivals.

- The number of vehicles is determined implicitly by the number of routes. Likewise, if a depot vertex dispatch routes then it is located.

3.1 The Multi-Depot Electric Vehicle Location Routing Problem with Time Windows and Battery Swapping

Sets:

$U D_{0} \quad$ Vertices of depots for the dispatch of vehicles.

$U D_{+1} \quad$ Vertices of depots for the arrival of vehicles.

UD Union of all vertices of depots for the dispatch and arrival of vehicles.

C Customer vertices.

$R \quad$ Special vertices of recharging stations.

$S K_{i} \quad$ Dummy vertices associated with vertex $i \in\{C \cup R \cup U D\}$.

$S \quad$ Union of all sets $S K_{i}$.

$V \quad$ Union of vertices of customers $C$ recharging stations $R$ and dummies $S$. 
$C_{0} \quad$ Union of vertices of customers $C$ and depots for dispatch $U D_{0}$.

$V_{0} \quad$ Union of vertices $i \in V$ and depots for dispatch $U D_{0}$.

$V_{+1} \quad$ Union of vertices $i \in V$ and depots for arrival $U D_{+1}$.

$V_{0+1} \quad$ Union of vertices $i \in V$ and vertices $i \in U D$.

Parameters:

$i d_{i} \quad$ Depot identification number. Each depot has a number assigned and this must be equal for both the dispatch vertices and the arrival vertices representing it, $i \in U D$.

$d i_{i j} \quad$ Minimum distance between $i \in V_{0}$ and $j \in V_{+1}$.

$t d_{i j} \quad$ Time of travel between vertex $i \in V_{0}$ and $j \in V_{+1}, t d_{i}=d i_{i j} v^{-1}$ where $v$ is the speed of the vehicle.

$s_{i} \quad$ Service time required at vertex $i \in C_{0}$.

$e_{i} \quad$ Earliest time of the time window of vertex $i \in V_{0+1}$.

$l_{i} \quad$ Latest time of the time window of vertex $i \in V_{0+1}$.

$p_{i} \quad$ Demand of vertex $i \in V_{0}$.

$D \quad$ Freight capacity of the vehicle.

$Q \quad$ Battery capacity.

ct $\quad$ Swapping time of battery.

$c \quad$ Consumption rate of energy per distance unit.

$o \quad$ Maximum number of recharging points to locate.

$L B \quad$ Lower bond for the number of vehicles that can leave a depot.

$U B \quad$ Upper bond for the number of vehicles that can leave a depot.

$M \quad$ Very large positive number.

Decision variables:

$x_{i j} \quad$ Binary variable equal to one if in a route the vehicle moves from the vertex $i \in V_{0}$ to $j \in V_{+1} \mid i \neq$ $j$. Zero otherwise.

$y_{i} \quad$ Binary variable equal to one if at vertex $i \in R$ a BSS is located. Zero otherwise.

$t_{i} \quad$ Moment of time in which a vehicle arrives at the vertex $i \in V_{0+1}$.

$d_{i} \quad$ Amount of freight left when the vehicle arrives at vertex $i \in V_{0+1}$.

$q_{i} \quad$ Amount of energy left in the battery when the vehicle arrives at vertex $i \in V_{0+1}$.

$u_{i} \quad$ Identification number of the depot $h \in U D_{0}$ from which the route starts that passes through the vertex $i \in V_{+1}$.

Objective function:

$$
\min \text { distance }=\sum_{i \in V_{0}} \sum_{j \in V_{+1} \mid i \neq j} d i_{i j} \cdot x_{i j}
$$

Constraints:

$$
\begin{array}{lr}
\sum_{j \in V_{++1} \mid i \neq j} x_{i j}=1 & \forall i \in C \\
\sum_{j \in V_{++1} \mid i \neq j} x_{i j} \leq 1 & \forall i \in\{R \cup S\} \\
\sum_{i \in V_{+1} \mid i \neq j} x_{j i}=\sum_{i \in V_{0} \mid i \neq j} x_{i j} & \forall j \in V \\
L B \leq \sum_{j \in V_{+1}} x_{i j} \leq U B & \forall i \in U D_{0} \\
\sum_{i \in R} y_{i} \leq o &
\end{array}
$$




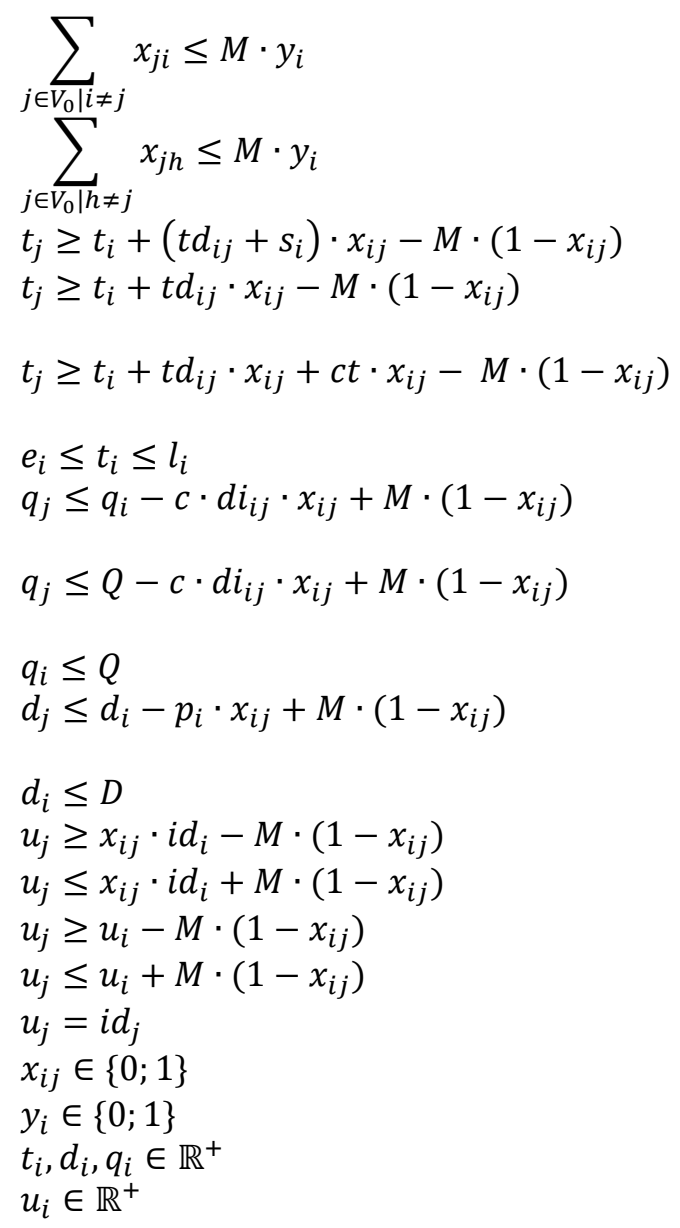

The constraints (2) - (5) guarantee the conformation of the routes. In (2) it is guaranteed that all the customer nodes are visited. In (3) it is established that the vertices of the charging stations and dummies can be visited once at most. In (4) the balance between input and output arcs is established and in (5) it is established that the number of arcs coming out of a depot is between the lower and upper bound; this restriction allows to minimize the number of vehicles if $U B$ is modified as a decision variable to be minimized. The constraints (6) - (8) are related to the location decisions of the charging stations. In (6) the number of stations to be located is limited; furthermore, this restriction allows to minimize the number of stations if $o$ is defined as a decision variable to optimize. On the other hand, restrictions (7) and (8) guarantee that if a recharging station is not located then the routes cannot pass through those nodes or their associated dummies. The constraints (9) - (12), allow to control the time of the vehicles in the routes, guaranteeing the compliance of the time windows and implicitly guaranteeing the non-conformation of subtours. In constraint (9) if a movement between the vertices $i$ and $j$ is performed, starting at a depot or customer, then the arrival time at $j$ is determined as the sum of the start time $t_{i}$, the displacement time to $j$ and the service time at $i$ (service time of zero for depots). Similarly, constraint (10) guarantees the time control for the customers' dummies but without considering the service time. The constraint (11) models the case in which a movement is performed from a recharging station and its dummy vertices, in which case in addition to the starting time at $i$ and the displacement time to $j$, the swapping time of the battery $c t$ is added. Finally, the constraint (12) guarantees the fulfillment of the time windows.

The restrictions (13) - (15) guarantee control over the battery power. In restriction (13) if a movement is made between the vertices $i$ and $j$ that initiates at a depot, a customer, or a customer dummy, it is guaranteed that the amount of energy at $j$ is less than that at $i$ minus that consumed in the displacement to $j$. In (14) if the movement is carried out from a recharging station, dummy vertex, or recharging station then the energy left in the battery that reaches at $j$ must be less than the total capacity of the battery $(Q)$ 
minus the energy consumed in the displacement to $j$. Finally, restriction (15) guarantees the maximum energy with which a vehicle can depart from a depot. The control over the cargo of merchandise is guaranteed with restrictions (16) and (17). In (16) it is guaranteed in the movements of the route that the merchandise delivered to customers is discounted, while in (17) the maximum quantity of merchandise with which a vehicle can start the route is defined. Finally, the restrictions (18) - (22) guarantee that the routes start and end at the same depots. This is achieved by defining an identifier number for each depot. Mark that share the vertex of dispatch $i \in U D_{0}$ and the one of arrival $k \in U D_{+1}$ that represent a certain depot (if $i$ and $k$ are associated to the same depot then $i d_{i}=i d_{k}$ ). With this feature, constraints (18) and (19) guarantee that when an arc is activated from a depot $i \in U D_{0}$ towards any other vertex $j \in V$, the variable $u_{j}$ takes the value of the identifier $i d_{i}$. Next, constraints (20) and (21) ensure that the variable $u_{i}$ of any vertex $i$ belonging to a route takes the value of the identifier number associated with the depot from which the route was dispatched. The restriction (22) then forces the routes to leave and arrive at the same depot. The no negativity condition of the decision variables is given by the constraints (23) - (26).

\subsection{The Multi-Depot Electric Vehicle Location Routing Problem with Time Windows and Partial Recharging}

The model holds the sets, parameters, objective function, and decision variables of the MDEVLRPTWBS with the following additions and changes:

\section{Parameters:}

$r \quad$ Energy charging rate per unit of time.

Variable decisions:

$y_{i} \quad$ Binary variable is equal to one if at the vertex $i \in\{C \cup R\}$ a recharging station is located. Zero otherwise. This variable replaces that of MDEVLRPTW-BS.

$w_{i} \quad$ Amount of energy to be charged at vertex $i \in V$.

Constraints:

Constraints (6) - (8), (10), (11), (13), (14) y (24) are eliminated, and the following restrictions are added to the model:

$$
\begin{aligned}
& \sum_{i \in\{C \cup R\}} y_{i} \leq o \\
& w_{i} \leq M \cdot y_{i} \\
& w_{h} \leq M \cdot y_{i} \\
& t_{j} \geq t_{i}+t d_{i j} \cdot x_{i j}+r \cdot w_{i}-M \cdot\left(1-x_{i j}\right) \\
& q_{j} \leq q_{i}-c \cdot d i_{i j} \cdot x_{i j}+M \cdot\left(1-x_{i j}\right) \\
& q_{j} \leq q_{i}+w_{i}-c \cdot d i_{i j} \cdot x_{i j}+M \cdot\left(1-x_{i j}\right) \\
& q_{i}+w_{i} \leq Q \\
& y_{i} \in\{0 ; 1\} \\
& w_{i} \in \mathbb{R}^{+}
\end{aligned}
$$

$$
\begin{array}{r}
\forall i \in\{C \cup R\} \\
\forall i \in\{C \cup R\} \\
\forall i \in\{C \cup R\}, h \\
\in S K_{i} \\
\forall i \in V, j \in V_{+1} \mid i \\
\neq j \quad \forall i \in U D_{0}, j \\
\in V_{+1} \mid i \neq j \\
\forall i \in V, j \in V_{+1} \mid i \\
\neq j \quad \forall i \in V \\
\forall i \in\{C \cup R\} \\
\forall i \in V
\end{array}
$$

The restriction (27) limits the number of charging stations to be located. The restrictions (28) and (29) guarantee that if a recharging station is not located, then energy cannot be charged at the associated vertex. Constraint (30) includes control over the time used to charge the battery in a conventional manner. 
The restrictions (31) and (32) model the control over the amount of energy in the vehicle's battery. The restriction (33) ensures the maximum capacity of the battery. Finally, constraints (34) and (35) guarantee non-negativity in the variables.

\subsection{The Multi-Depot Electric Vehicle Location Routing Problem with Time Windows Battery Swapping and Partial Recharging}

The model holds the sets, parameters, objective function, and decision variables of the MDEVLRPTW$\mathrm{BS}$ with the additions and changes described in the MDEVLRPTW-PR.

Only the decision variable $w_{i}$ is changed to represent the amount of energy to be charged at vertex $i \in$ $\left\{C \cup S K_{h \in C}\right\}$.

Constraints:

Constraints (6), (10), (13) y (24) are eliminated, constraints (27) and (34) are added in addition to the following constraints:

$$
\begin{aligned}
& w_{i} \leq M \cdot y_{i} \\
& w_{h} \leq M \cdot y_{i} \\
& t_{j} \geq t_{i}+t d_{i j} \cdot x_{i j}+r \cdot w_{i}-M \cdot\left(1-x_{i j}\right) \\
& q_{j} \leq q_{i}-c \cdot d i_{i j} \cdot x_{i j}+M \cdot\left(1-x_{i j}\right) \\
& q_{j} \leq q_{i}+w_{i}-c \cdot d i_{i j} \cdot x_{i j}+M \cdot\left(1-x_{i j}\right) \\
& q_{i}+w_{i} \leq Q \\
& w_{i} \in \mathbb{R}^{+}
\end{aligned}
$$

$$
\forall i \in C
$$$$
\forall i \in C, h \in S K_{i}
$$$$
\forall i
$$$$
\in\left\{C \cup S K_{h \in C}\right\}
$$$$
j \in V_{+1} \mid i \neq j
$$$$
\forall i \in\left\{U D_{0}\right\}, j
$$$$
\in V_{+1} \mid i \neq j
$$$$
\in\left\{C \cup S K_{h \in C}\right\},
$$$$
j \in V_{+1} \mid i \neq j
$$$$
\forall i
$$$$
\in\left\{C \cup S K_{h \in C}\right\}
$$$$
\in\left\{C \cup S K_{h \in C}\right\}
$$

Constraints (36) and (37) ensure that if a charging station is not located at customer vertices or its dummies, then no charging can be done at those vertices. Constraint (38) allows for considering conventional charging in the time control at customers and associated dummies vertices (when movements are made from these vertices). Constraint (41) ensures that the vehicles' energy does not surpass the battery capacity. Constraint (42) is necessary to ensure non-negativity in the decision variables.

\subsection{Solution strategy}

The optimization models were implemented in $\mathrm{C}++$ and the experiments were executed on an Intel Core i7 processor with OS Ubuntu 15.04 LTS and memory of 8 GB. CPLEX 12.5 was used to solve the models. The time limit for solving the instances was set to 28800 seconds. In addition, to improve the computational times it was considered that mobilizations between vertices $i \in V \backslash\{S\}$ and the associated dummy vertices $S K_{i}$ have no sense. So, the pre-processing constraints (43) and (44) were used:

$$
\begin{array}{rr}
x_{i j}=0 & \forall i \in V \backslash\{S\}, \\
x_{j i}=0 & j \in S K_{i} \mid i \neq j \\
\forall j \in V \backslash\{S\},
\end{array}
$$


The experiments were executed using instances of five, ten, and fifteen customers proposed by Schiffer and Walther (2017). These instances were modified to obtain a multi-depot problem, in that it is not found in the literature instances of this type for EVs. The modification was the random addition of a vertex where a depot can be located. This was done by ensuring that the coordinates were between the minimum and maximum distance from the original instances. These tests can be downloaded from http://academia.utp.edu.co/planeamiento/?p=3561.

Apart from this, it was defined that each vertex $\mathrm{i} \in\{C \cup R \cup U D\}$ had a single dummy vertex. This is due to the small size of the resolved instances. Additionally, the following parameters required adjusting, with values to execute the experiments:

ct was defined as ten percent of the total battery charge time by conventional means.

$o \quad$ was defined equal to the number of customers' nodes and charging stations.

$L B \quad$ was defined using the bin packing problem strategy proposed by Schiffer and Walther (2017). This limit improves computational times.

$U B \quad$ was defined as equal to the number vertices of customers $(C)$ and special charging stations $(R)$.

$M \quad$ was defined as $2 \cdot\left(Q+C+l_{U D_{0[1]}}\right)$, in which $U D_{0_{[1]}}$ is the first element of $U D_{0}$.

The parameters $o$ and $U B$ do not restrict either the number of stations required or the number of vehicles used in the depots; however, if they were postulated as decision variables and defined as objective functions in which they are minimized, the mathematical structures proposed would allow to orient the model to the minimization of the number of vehicles and the number of stations, respectively. These parameters were not defined with values because the original instances do not have them and defining an arbitrary value could have skewed the results.

\section{Computational results, analysis and discussion}

The results obtained by the models are shown in Table 1. The characteristics of interest of the network: the depots and stations to be located, as well as the routes of operation, are shown in the results.

A particularity of these results is that for the case of BS, the distance is always greater than for the other two models. In the case of BSPR and PR, the optimization objective is the same for almost all instances. On the other hand, only in the case of instances rc103-15 and rc108-15 the PR strategy shows a better result; however, these results are not optimal, so it is not accurate to conclude against the results shown by these instances. Despite this behavior in the results of the objective function of the BSPR and PR models, it should be noted that the design of the network differs in the number of charging stations located at customers nodes; behavior that can be seen in Fig. 1 (stations marked with squares).

As shown in Fig. 1, instance c103-5 presents the same routes for both models, so the minimum distance traveled is the same; however, each model differs in the number of stations located. This is because the models do not consider the cost associated with the location of the stations and this causes the existence of several ways of locating the stations and at the same time, guarantees the autonomy of the vehicle. It is evident then that the problem presents multiple solutions. On the other hand, the results show that the number of depots and vehicles used is the same for the case of BSPR and PR. In addition, the number of BSS used in the case of BSPR tends to zero. This suggests that the conventional charge strategy generates better results in the evaluated instances; however, in three instances this behavior was not fulfilled (r1055, c101-10 and r209-15) since regardless of the type of station, a BSS station is always located. This is because for the fulfillment of the routes of these instances, the localized stations are the only possible point of recharge on the road before running out of power. From what is evident, the influence of the geospatial position on the decisions of location of stations. 

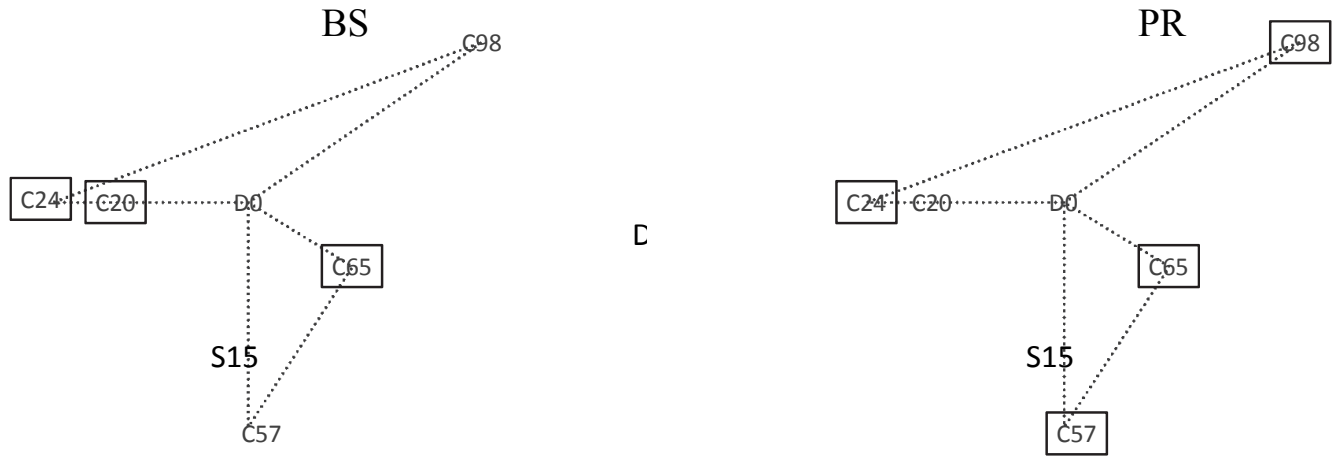

[

Fig. 1. Routes of instance c103-5

Table 1

Results for the MDEVLRPTW-BS, MDEVLRPTW-BSPR, and the MDEVLRPTW-PR, minimizing the overall distance traveled

\begin{tabular}{|c|c|c|c|c|c|c|c|c|c|c|c|c|c|c|c|c|c|}
\hline \multirow[b]{2}{*}{ inst. } & \multirow[b]{2}{*}{$\mathrm{dp}$. } & \multicolumn{4}{|c|}{ MDEVLRPTW-BS } & \multicolumn{6}{|c|}{ MDEVLRPTW-BSPR } & \multicolumn{6}{|c|}{ MDEVLRPTW-PR } \\
\hline & & vh. & dist. & st. & time & $\mathrm{dp}$. & vh. & dist. & cst. & st. & time & dp. & vh. & dist. & cst. & st. & time \\
\hline c101-5 & 2 & 4 & 238.62 & 1 & 0.30 & 2 & 3 & 229.31 & 3 & 0 & 0.44 & 2 & 3 & 229.31 & 4 & 0 & 0.42 \\
\hline c103-5 & 1 & 3 & 165.67 & 0 & 0.55 & 1 & 2 & 161.26 & 3 & 0 & 0.32 & 1 & 2 & 161.26 & 4 & 0 & 0.48 \\
\hline c206-5 & 2 & 4 & 234.31 & 1 & 2.14 & 2 & 3 & 218.58 & 5 & 0 & 0.86 & 2 & 3 & 218.58 & 5 & 0 & 4.53 \\
\hline c208-5 & 1 & 2 & 129.13 & 1 & 1.09 & 1 & 1 & 127.86 & 4 & 0 & 1.57 & 1 & 1 & 127.86 & 5 & 0 & 1.53 \\
\hline r104-5 & 2 & 2 & 118.51 & 1 & 0.61 & 2 & 2 & 118.27 & 5 & 0 & 0.90 & 2 & 2 & 118.27 & 4 & 0 & 0.63 \\
\hline r105-5 & 2 & 2 & 104.70 & 1 & 0.18 & 2 & 2 & 102.83 & 4 & 1 & 0.18 & 2 & 2 & 102.83 & 4 & 1 & 0.21 \\
\hline r $202-5$ & 1 & 1 & 126.90 & 3 & 1.85 & 1 & 1 & 124.39 & 4 & 0 & 0.88 & 1 & 1 & 124.39 & 4 & 0 & 5.77 \\
\hline r203-5 & 2 & 2 & 167.33 & 2 & 0.63 & 2 & 2 & 161.15 & 3 & 0 & 0.61 & 2 & 2 & 161.15 & 5 & 0 & 0.69 \\
\hline rc $105-5$ & 2 & 3 & 185.72 & 1 & 0.66 & 2 & 3 & 179.50 & 4 & 0 & 0.48 & 2 & 3 & 179.50 & 4 & 0 & 0.79 \\
\hline rc108-5 & 2 & 2 & 242.10 & 2 & 7.09 & 2 & 2 & 238.03 & 4 & 0 & 4.12 & 2 & 2 & 238.03 & 5 & 0 & 20.78 \\
\hline rc204-5 & 1 & 1 & 176.39 & 3 & 38.15 & 2 & 2 & 169.27 & 5 & 0 & 34.94 & 2 & 2 & 169.27 & 5 & 0 & 31.64 \\
\hline rc208-5 & 1 & 1 & 167.98 & 2 & 9.57 & 1 & 1 & 162.67 & 5 & 0 & 2.63 & 1 & 1 & 162.67 & 5 & 0 & 11.91 \\
\hline c101-10 & 2 & 4 & 331.10 & 3 & 582.72 & 2 & 3 & 316.21 & 9 & 1 & 15.08 & 2 & 3 & 316.21 & 10 & 1 & 39.63 \\
\hline c104-10 & 2 & 2 & 243.37 & 3 & 41.99 & 1 & 1 & 234.22 & 8 & 0 & 2.21 & 1 & 1 & 234.22 & 9 & 0 & 12.43 \\
\hline c202-10 & 2 & 3 & 227.25 & 2 & 27.45 & 2 & 2 & 217.20 & 6 & 0 & 1.44 & 2 & 2 & 217.20 & 9 & 0 & 2.34 \\
\hline c205-10 & 2 & 3 & 204.44 & 1 & 1.64 & 2 & 3 & 204.43 & 7 & 0 & 1.20 & 2 & 3 & 204.43 & 7 & 0 & 1.86 \\
\hline r102-10 & 2 & 3 & 232.37 & 3 & 28.58 & 2 & 3 & 224.32 & 8 & 0 & 9.41 & 2 & 3 & 224.32 & 7 & 0 & 21.64 \\
\hline r103-10 & 2 & 3 & 193.41 & 2 & 9747.56 & 1 & 2 & 188.67 & 9 & 0 & 4922.05 & 1 & 2 & 188.67 & 9 & 0 & 8581.20 \\
\hline r201-10 & 2 & 4 & 206.74 & 2 & 16.06 & 2 & 3 & 195.06 & 9 & 0 & 2.87 & 2 & 3 & 195.06 & 7 & 0 & 7.80 \\
\hline r203-10 & 1 & 1 & 216.99 & 4 & 10880.50 & 1 & 1 & 209.27 & 7 & 0 & 277.96 & 1 & 1 & 209.27 & 9 & 0 & 4171.44 \\
\hline rc102-10 & 2 & 5 & 386.96 & 1 & 18.63 & 2 & 3 & 362.67 & 9 & 0 & 2.88 & 2 & 3 & 362.67 & 9 & 0 & 5.92 \\
\hline rc108-10 & 2 & 4 & 312.04 & 2 & 89.41 & 2 & 3 & 287.89 & 9 & 0 & 9.17 & 2 & 3 & 287.89 & 8 & 0 & 36.57 \\
\hline rc201-10 & 2 & 4 & 298.00 & 1 & 94.39 & 2 & 4 & 292.34 & 8 & 0 & 197.46 & 2 & 4 & 292.34 & 9 & 0 & 226.18 \\
\hline rc205-10 & 2 & 3 & 290.87 & 2 & 3.93 & 2 & 3 & 290.52 & 9 & 0 & 2.60 & 2 & 3 & 290.52 & 6 & 0 & 2.28 \\
\hline c103-15 & 2 & 3 & 320.17 & 2 & 28800.00 & 2 & 3 & 315.56 & 9 & 0 & 28800.00 & 2 & 3 & 315.56 & 12 & 0 & 28800.00 \\
\hline c106-15 & 2 & 4 & 261.20 & 1 & 68.45 & 2 & 4 & 255.05 & 13 & 0 & 7.89 & 2 & 4 & 255.05 & 14 & 0 & 26.28 \\
\hline c202-15 & 2 & 3 & 353.75 & 4 & 1104.17 & 2 & 3 & 341.34 & 11 & 0 & 476.33 & 2 & 3 & 341.34 & 11 & 0 & 946.80 \\
\hline c208-15 & 1 & 2 & 300.55 & 3 & 635.45 & 1 & 2 & 293.89 & 12 & 0 & 661.41 & 1 & 2 & 293.89 & 14 & 0 & 341.45 \\
\hline r102-15 & 2 & 5 & 335.47 & 3 & 2293.45 & 2 & 5 & 307.53 & 12 & 0 & 170.65 & 2 & 5 & 307.53 & 10 & 0 & 149.61 \\
\hline r105-15 & 2 & 4 & 289.00 & 3 & 17.43 & 2 & 4 & 288.34 & 10 & 0 & 6.33 & 2 & 4 & 288.34 & 12 & 0 & 13.18 \\
\hline r202-15 & 2 & 3 & 323.18 & 4 & 5422.84 & 2 & 3 & 322.51 & 11 & 0 & 17330.10 & 2 & 3 & 322.51 & 14 & 0 & 28800.00 \\
\hline r209-15 & 1 & 2 & 274.34 & 4 & 1258.39 & 2 & 2 & 254.63 & 10 & 1 & 112.33 & 2 & 2 & 254.63 & 15 & 1 & 61.00 \\
\hline rc103-15 & 2 & 5 & 354.26 & 3 & 28800.00 & 2 & 5 & 349.38 & 13 & 0 & 28800.00 & 2 & 4 & 347.21 & 10 & 0 & 28800.00 \\
\hline rc108-15 & 1 & 3 & 372.54 & 4 & 28800.00 & 2 & 3 & 362.47 & 14 & 0 & 28800.00 & 1 & 3 & 358.78 & 14 & 0 & 28800.00 \\
\hline rc202-15 & 2 & 3 & 370.76 & 3 & 800.80 & 2 & 3 & 363.45 & 13 & 0 & 346.53 & 2 & 3 & 363.45 & 13 & 0 & 516.25 \\
\hline rc204-15 & 1 & 2 & 310.58 & 4 & 28800.00 & 1 & 2 & 304.33 & 12 & 0 & 28800.00 & 1 & 2 & 304.33 & 14 & 0 & 28800.00 \\
\hline
\end{tabular}

Abbreviations hold as follows: inst. $=$ test instance; $\mathrm{dp} .=$ number of depot used; vh. $=$ number of vehicles used; dist. $=$ overall traveled distance; cst. = number of charging stations at customers; st. = number of specialized charging stations used-standard recharging stations for PR and swapping stations for BS and BSPR; time = computational time [s]. 
With respect to the computational times of solution of the models, the results do not show a clear pattern of behavior that allows to differentiate one model from another. What is clear is that the results are limited by the size of the instances. Only instances of up to fifteen customers were solved, and some of them could not be solved optimally (Table 2).

Table 2

Gap values of the non-optimal instances

\begin{tabular}{llrr}
\hline inst. & MDEVLRPTW-BS & MDEVLRPTW-BSPR & MDEVLRPTW-PR \\
\hline c103-15 & 0.0413 & 0.0363 & 0.0633 \\
r202-15 & & & 0.0328 \\
rc103-15 & 0.1140 & 0.1871 & 0.0989 \\
rc108-15 & 0.2651 & 0.2193 & 0.2184 \\
rc204-15 & 0.1987 & 0.1959 & 0.1914 \\
\hline
\end{tabular}

\section{Conclusions}

In this article, three models of location and routing for EVs were proposed considering classic restrictions in the literature and extending it to the case of multiple depots. Two types of charge approach were considered: BSS, and conventional charging stations. The results obtained validate the proposed model to solve small-scale problems in an optimal way. It was found that the proposed modeling approach allowed to compare the two strategies considering battery charge and with this, determined the best design for the EVs' logistics network.

On the other hand, the results showed that the costs of installation and operation of the network impact significantly on the design decisions of the network; so this is an aspect that should be considered for real design applications. Apart from this, it was found that in order to achieve a better design in this type of problem, in future it is necessary to consider work elements that allow deciding between different types of technology for the stations when the geospatial conditions of the network force locating a recharging station. Future work can be oriented to the design of solution strategies for large-scale problems, as well as tests with real application data for the treated case.

\section{Acknowledgements}

The authors of this paper would like to thank Colciencias and Universidad Tecnológica de Pereira for their financial support under call for research 706 of 2015, which we gratefully acknowledge.

\section{References}

Afroditi, A., Boile, M., Theofanis, S., Sdoukopoulos, E., \& Margaritis, D. (2014). Electric vehicle routing problem with industry constraints: trends and insights for future research. Transportation Research Procedia, 3, 452-459.

Barco, J., Guerra, A., Muñoz, L., \& Quijano, N. (2013). Optimal routing and scheduling of charge for electric vehicles: Case study. arXiv preprint arXiv:1310.0145.

Cavadas, J., de Almeida Correia, G. H., \& Gouveia, J. (2015). A MIP model for locating slow-charging stations for electric vehicles in urban areas accounting for driver tours. Transportation Research Part E: Logistics and Transportation Review, 75, 188-201.

Conrad, R. G., \& Figliozzi, M. A. (2011, January). The recharging vehicle routing problem. In IIE Annual Conference. Proceedings (p. 1). Institute of Industrial Engineers-Publisher.

Desaulniers, G., Errico, F., Irnich, S., \& Schneider, M. (2016). Exact algorithms for electric vehiclerouting problems with time windows. Operations Research, 64(6), 1388-1405.

Ding, N., Batta, R., \& Kwon, C. (2015). Conflict-Free Electric Vehicle Routing Problem with Capacitated Charging Stations and Partial Recharge. 
Drexl, M., \& Schneider, M. (2014). A survey of the standard location-routing problem. Darmstadt Technical University, Department of Business Administration, Economics and Law, Institute for Business Studies (BWL).

Drexl, M., \& Schneider, M. (2015). A survey of variants and extensions of the location-routing problem. European Journal of Operational Research, 241(2), 283-308.

Erdoğan, S., \& Miller-Hooks, E. (2012). A green vehicle routing problem. Transportation Research Part E: Logistics and Transportation Review, 48(1), 100-114.

Felipe, A., Ortuño, M. T., Righini, G., \& Tirado, G. (2014). A heuristic approach for the green vehicle routing problem with multiple technologies and partial recharges. Transportation Research Part E: Logistics and Transportation Review, 71, 111-128.

Goeke, D., \& Schneider, M. (2015). Routing a mixed fleet of electric and conventional vehicles. European Journal of Operational Research, 245(1), 81-99.

Hiermann, G., Puchinger, J., Ropke, S., \& Hartl, R. F. (2016). The electric fleet size and mix vehicle routing problem with time windows and recharging stations. European Journal of Operational Research, 252(3), 995-1018.

Keskin, M., \& Çatay, B. (2016). Partial recharge strategies for the electric vehicle routing problem with time windows. Transportation Research Part C: Emerging Technologies, 65, 111-127.

Li-ying, W., \& Yuan-bin, S. (2015). Multiple Charging Station Location-Routing Problem with Time Window of Electric Vehicle. Journal of Engineering Science and Technology Review, 8(5), 190-201.

Montoya, A., Guéret, C., Mendoza, J. E., \& Villegas, J. G. (2016). A hybrid metaheuristic for the electric vehicle routing problem with partial charging and nonlinear charging function.

Omidvar, A., \& Tavakkoli-Moghaddam, R. (2012, September). Sustainable vehicle routing: Strategies for congestion management and refueling scheduling. In Energy Conference and Exhibition (ENERGYCON), 2012 IEEE International (pp. 1089-1094). IEEE.

Prodhon, C., \& Prins, C. (2014). A survey of recent research on location-routing problems. European Journal of Operational Research, 238(1), 1-17.

Schiffer, M., \& Walther, G. (2016). An adaptive large neighbourhood search for the location routing problem with intraroute facilities. Working paper OM-01.

Schiffer, M., \& Walther, G. (2017). The electric location routing problem with time windows and partial recharging. European Journal of Operational Research.

Schneider, M., Stenger, A., \& Goeke, D. (2014). The electric vehicle-routing problem with time windows and recharging stations. Transportation Science, 48(4), 500-520.

Touati-Moungla, N., \& Jost, V. (2012). Combinatorial optimization for electric vehicles management. Journal of Energy and Power Engineering, 6(5).

UPS (2013). UPS to rollout fleet of electric vehicles in California.

Wang, Y. W. (2008). Locating battery exchange stations to serve tourism transport: A note. Transportation Research Part D: Transport and Environment, 13(3), 193-197.

Wang, Y. W., \& Lin, C. C. (2009). Locating road-vehicle refueling stations. Transportation Research Part E: Logistics and Transportation Review, 45(5), 821-829.

Wang, Y. W., \& Wang, C. R. (2010). Locating passenger vehicle refueling stations. Transportation Research Part E: Logistics and Transportation Review, 46(5), 791-801.

Worley, O., Klabjan, D., \& Sweda, T. M. (2012, March). Simultaneous vehicle routing and charging station siting for commercial electric vehicles. In Electric Vehicle Conference (IEVC), 2012 IEEE International (pp. 1-3). IEEE.

Yang, J., \& Sun, H. (2015). Battery swap station location-routing problem with capacitated electric vehicles. Computers \& Operations Research, 55, 217-232.

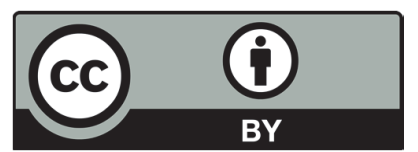

(C) 2017 by the authors; licensee Growing Science, Canada. This is an open access article distributed under the terms and conditions of the Creative Commons Attribution (CCBY) license (http://creativecommons.org/licenses/by/4.0/). 